\title{
EVALUACIÓN DE CUATRO PATRONES PARA INJERTOS DE GUAYABA (Psidium guajava L. $)^{1}$
}

\author{
Antonio Bogantes-Arias ${ }^{2}$,Eric Mora-Newcomer ${ }^{3}$
}

\section{RESUMEN}

Evaluación de cuatro patrones para injertos de guayaba (Psidium guajava $\mathbf{L}$.). El trabajo se realizó en la Estación Experimental Los Diamantes del INTA entre el 2004 y el 2007, con el objetivo de determinar el efecto de varios patrones en la injertación de la guayaba. Se evaluaron cuatro patrones: tres de cas (Psidium friedrichsthalianum), y uno de guayaba, sobre el crecimiento y desarrollo de un clon experimental de guayaba (Psidium guajava). En la etapa de vivero, el prendimiento fue de un $100 \%$ para los patrones guayaba y "cas brasileño", mientras que para los tratamientos con "arrayán" y "cas criollo" fue de un 90\%. El crecimiento vegetativo inicial fue significativamente mayor en el testigo. En el campo, se observó la misma tendencia de mayor crecimiento en el tratamiento de guayaba como patrón. También, se observó un claro efecto enanizante del patrón arrayán, mientras que los patrones "cas brasileño" y "cas criollo" mostraron un crecimiento intermedio. El número y peso de la fruta fue superior en el tratamiento de guayaba como patrón y no hubo diferencias entre los tipos de cas. Con el fin de evaluar la susceptibilidad de los patrones a nematodos en plantas adultas, se incluyó un testigo adicional de guayaba al que se le aplicó nematicida en campo. Los resultados indican que los nematodos Meloidogyne sp. y Pratylenchus sp. lograron colonizar las raíces de los tratamientos de guayaba con y sin nematicida, así como del cas brasileño. No se detectaron nematodos en las raíces de los patrones cas criollo ni de arrayán.

Palabras clave: Cas arrayán, cas brasileño, nematodos, Psidium guajava.

\begin{abstract}
Evaluation of four rootstocks for graft in guava (Psidium guajava L.). The work was conducted at Estación Experimental Los Diamantes, INTA, from 2004 to 2007, in order to determine the effectiveness of various rootstocks on guava grafting. Four rootstocks were evaluated: three different Psidium friedrichsthalianum (cas) rootstocks and one Psidium guajava (guava) rootstock, on the growth and development of a guava experimental clone. In the nursery, $100 \%$ successful grafts were obtained with guava and "brazilian" cas rootstocks, while success with "arrayán" and "cas criollo" was $90 \%$. Initial vegetative growth was significantly higher in the guava rootstock treatment. In the field, this treatment also showed more vigorous growth. "Arrayán" rootstocks clearly showed a strong dwarfing effect, while the "Brazilian cas" and the "cas Criollo" rootstocks exhibited an intermediate effect on vegetative vigor. Number and weight of fruits was higher when guava was used as a rootstock, and no differences were observed with cas rootstocks. In order to evaluate the susceptibility of the rootstocks to nematodes of adult plants, an additional control treatment was included in the field, which consisted of the application of nematicide to the guava rootstock treatment. The results indicate that Meloidogyne sp. and Pratylenchus sp. were able to colonize the roots of the guava rootstocks with and without nematicide, as well as roots of "Brazilian cas". No nematodes were detected in the roots of the "cas "criollo" and "Arrayán" rootstocks.
\end{abstract}

Key words: Cas "arrayán", Brazilian cas, nematodes, Psidium guajava.

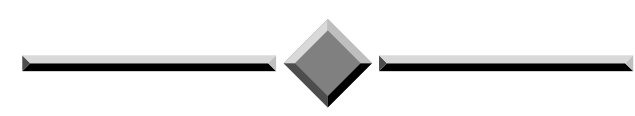

\footnotetext{
Recibido: 9 de julio, 2009. Aceptado: 14 de junio, 2010. Este trabajo forma parte de un proyecto conjunto entre el Instituto Nacional de Innovación y Transferencia en Tecnología Agropecuaria (INTA) y la Universidad de Costa Rica (UCR).

2 Instituto Nacional de Innovación y Transferencia en Tecnología Agropecuaria - Ministerio de Agricultura y Ganadería (INTA-MAG). San José, Costa Rica. Tel. (506) 710-7852. bogantesa@ costarricense.cr

3 Estación Experimental Fabio Baudrit Moreno. Universidad de Costa Rica. (506) 433-9132. emoran@racsa.co.cr
} 


\section{INTRODUCCIÓN}

La guayaba (Psidium guajava L.) es una planta originaria de Mesoamérica y se desarrolla ampliamente en los trópicos donde enriquece la dieta de cientos de millones de personas. La fruta se distingue por su sabroso y fresco aroma; un alto contenido de vitamina C (200-500 mg/100 de fruta) y apreciable cantidad de vitamina A (250-400 i.u./100 g de fruta); posee ácido ascórbico y hierro (Perales et al. 2005). En México ocupa el doceavo lugar entre las principales especies $\mathrm{y}$ variedades de frutas que se explotan comercialmente con más de 20000 ha reportadas en 1997 (González et al. 2002).

En Costa Rica, se usa principalmente para la industria, en jaleas o mermeladas para lo cual se recolecta fruta en árboles silvestres de cercas o potreros. A pesar de su potencial para exportación como fruta de mesa las áreas comerciales de siembra son escasas y obedece a plantaciones aisladas de la variedad taiwanesa "TaiKuo-Bar" de pulpa blanca (Román y Wann 1999), la cual es muy susceptible a Ceratitis sp.

La pulpa roja es un atractivo para el mercado nacional, motivo por el cual dentro del trabajo conjunto entre la Universidad de Costa Rica (UCR) y el Instituto de Innovación y Transferencia de Tecnología (INTA) se han cruzado variedades introducidas con materiales criollos, para buscar guayaba de ciertas características, tanto para mesa como para industria. Los primeros resultados han sido la obtención de dos materiales (B8-27 de pulpa blanca y R1-22 rosada) con buenas características para mesa, a los cuales, se les debe mantener sus características, para su validación.

La propagación asexual del guayabo permite lograr la reproducción de individuos idénticos, a partir de porciones vegetativas de plantas que tengan capacidad de regeneración (Perales et al. 2005).

La reproducción asexual en guayaba, se hace en forma común por estacas, acodos, injertos o in vitro (Dantas et al. 1999, González et al. 2002, Pérez et al. 2002, Ramírez et al. 2003, Vilchez et al. 2004, Perales et al. 2005). En esos casos los porcentajes de germinación son bajos y requieren de un mayor costo.

Las técnicas de injertación probadas en guayaba han sido el de yema en parche o " $\mathrm{T}$ " invertida y el de púa o vareta (corte con tres a cuatro yemas) en hendidura lateral (Perales et al. 2005). Los autores de este estudio en pruebas preliminares (datos sin publicar), comprobaron que es viable el injerto en guayaba con el uso de la vareta envuelta en plástico y la técnica de injertación con hendidura terminal en el patrón. El uso de la misma guayaba como portainjerto (patrón), sin una selección previa, trae como consecuencia negativa, susceptiblidad a nematodos, principalmente Meloidogyne incognita (Suárez y Rosales 1998).

Un patrón ideal para la injertación sería aquel que se adapte al clima y al suelo, debería ser tolerante a los patógenos e inducir alta producción y calidad de fruta, y árboles de porte pequeño, que facilite las operaciones de cosecha y fumigación (Monteverde 1989).

Las premisas bajo las cuales se desenvuelve la fruticultura moderna son: adaptar la planta al espacio y no a la inversa, así como modificar o alterar sus fenofases. Los árboles caracterizados por presentar a lo largo de su vida útil un bajo y mediano porte, debido a la presencia de entrenudos cortos (braquitísmo), constituyen una alternativa como patrones e interpatrones para inducir o reducir el tamaño de las plantas y mayores densidades de población (Avilán et al. 2003).

La elección del mejor patrón representa uno de los problemas más complejos de la fruticultura. Los estudios para la selección de patrones en manzano, conocidos mundialmente por las siglas EM, permitieron su desarrollo y que en la actualidad cuentan con materiales para solventar parte de la problemática de ese cultivo (Avilán 2007).

Este trabajo, se planeó con el objetivo de determinar el efecto de patrones en la injertación de la guayaba.

\section{MATERIALES Y MÉTODOS}

El estudio se realizó entre abril del 2004 y junio del 2007 en la Estación Experimental Los Diamantes del Ministerio de Agricultura y Ganadería, ubicada en Guápiles, cantón Pococí, provincia de Limón; a una altitud de $249 \mathrm{msnm}$, con una temperatura mínima promedio de $24{ }^{\circ} \mathrm{C}$ y una máxima promedio de $29^{\circ} \mathrm{C}$. La precipitación promedio anual es de $4.500 \mathrm{~mm}$. El trabajo consistió de dos etapas, la primera en vivero y la segunda en campo.

\section{Etapa de vivero}

La etapa de vivero se hizo dentro de un invernadero con zinc plástico y transparente. Se evaluaron los siguientes patrones: 
1. Guayaba criolla (Psidium guajava)

2. Cas criollo (Psidium friedrichsthalianum)

3. Cas "brasileño" (Psidium friedrichsthalianum)

4. Cas "arrayán" (Psidium friedrichsthalianum)

La semilla de guayaba criolla, cas criollo y cas brasileño, fue recolectada en la Estación Experimental Los Diamantes. El cas "brasileño" fue introducido de Sur América en época desconocida. El cas conocido como "arrayán" (introducción CAT 9962) proviene del banco de germoplasma de la Unidad de Recursos Fitogenéticos del Centro Agronómico Tropical de Investigación y Enseñanza (CATIE) en Turrialba.

Los patrones se sembraron en bolsas de polietileno negro de $20 \mathrm{~cm}$ de fondo por $30 \mathrm{~cm}$ de alto con una mezcla $2: 1(\mathrm{vol} / \mathrm{vol})$ de suelo y un compost a base de cachaza de caña de azúcar que proviene de ingenio. Los injertos se hicieron sobre patrones que medían alrededor de $1,0 \mathrm{~cm}$ de grosor en la parte media del tallo. El material a injertar consistió en cortes terminales con tres o cuatro yemas, conocidas como varetas o púas. Estas varetas injertadas sobre los patrones fueron de la selección R9-33, que es un material experimental, homogéneo, promisorio y preseleccionado como resultado de un proyecto de mejora genética que se realiza entre la Universidad de Costa Rica y el Instituto de Innovación y Transferencia Tecnológica de Costa Rica (INTA). Las púas se cortaron de ramas seleccionadas y despuntadas dos semanas antes de su uso. Se aplicó la técnica del injerto de vareta, con hendidura terminal en el patrón.

Los tratamientos o injertos fueron distribuidos en un diseño completamente al azar con diez repeticiones.

\section{Variables evaluadas}

Se evaluó el porcentaje de varetas con brotes u hojas (prendimiento) a los 19 y 26 días después del injerto (ddi). El crecimiento del injerto se midió con base en el número de ramas, longitud y grosor $(\mathrm{cm})$ de la rama más larga en el injerto a los 54 y 75 ddi y la biomasa del patrón se obtuvo con base en el peso fresco (g) de las ramas o brotes podados al patrón (conocidas como "chupones"), hasta los 75 ddi.

Se hizo un análisis de varianza para las variables crecimiento del injerto y biomasa del patrón. En los casos en los que se determinaron diferencias entre tratamientos se hizo separación de medias por Duncan.

\section{Plantación definitiva}

Los árboles de guayaba R9-33, con 75 días de edad, originados de las varetas injertadas sobre los diferentes patrones, fueron sembrados en campo a una distancia de siembra de $3 \times 3 \mathrm{~m}$. Los tratamientos fueron los siguientes:

1. Guayaba criolla (Psidium guajava)

2. Guayaba criolla (Psidium guajava) + Nematicida

3. Cas criollo (Psidium friedrichsthalianum)

4. Cas "brasileño" (Psidium friedrichsthalianum)

5. Cas "arrayán" (Psidium friedrichsthalianum))

Fueron los mismos patrones de la etapa de vivero con un tratamiento adicional que consistió en guayaba R9-33 injertado sobre patrón de guayaba criolla pero tratado con nematicida, carbofurán $10 \mathrm{~g}$ de producto comercial por planta, a la siembra, a los seis y doce meses de edad.

Los tratamientos se sembraron con un diseño completamente al azar con diez repeticiones (un árbol por repetición) en un lote cuya totalidad, había sido sembrado con guayaba durante 20 años atrás.

Las variables evaluadas fueron:

1. Biomasa en el patrón: Peso fresco (g) de las ramas podadas al patrón (conocidas como "chupones") a los 50, 90, 135 días después de la siembra (dds).

2. Crecimiento del injerto: Número de ramas y longitud $(\mathrm{cm})$ acumulada de las ramas (sumatoria de la longitud de todas) en el injerto 50, 90, 135 dds.

3. Número de flores y de frutos en el injerto a los 135 y $250 \mathrm{dds}$.

4. Peso de frutos a los 250 y 300 dds.

5. Nematodos en raíz: Cantidad de nematodos en 100 g de raíz a los 600 dds.

Se hizo un análisis de varianza (ANDEVA) para las variables, excepto al porcentaje de varetas con brotes y hojas. En los casos en los que se determinaron diferencias entre tratamientos se hizo separación de medias por Duncan. Debido a problemas en la disponibilidad de fruta por merodeo (hurto), la variable peso de fruto no fue considerada para el ANDEVA. Con la cantidad de fruta colectada se establecieron los promedios, desviación estándar, y número de muestra por tratamiento. El ANDEVA para la variable nematodos 
no consideró los tratamientos cas criollo y cas arrayán porque en todas sus repeticiones la población fue cero. En los restantes tres tratamientos los datos fueron transformados a raíz de x para su análisis de varianza. Los resultados se muestran con los datos originales.

Todos los análisis fueron realizados utilizando el paquete estadístico del sistema SAS (López y López 1995).

\section{RESULTADOS Y DISCUSIÓN}

En el cas brasileño la inserción de las ramas fue de un ángulo muy agudo y con mucha rama secundaria, el tallo principal se abre con facilidad a la hora de hacer la hendidura para el injerto terminal y tiene los entrenudos muy cortos, lo cual dificulta un poco el amarre o encintado de la vareta. La guayaba es un patrón de menos ramas, más suculento en su tallo y fácil de abrir para insertar la vareta. El arrayán posee bastantes ramas, también tiene los entrenudos cortos y es semejante al cas criollo con sus ramas extendidas, casi a 90 grados con respecto al tallo principal, arquitectura que facilita la injertación y el amarre.

\section{Brotación de las varetas}

En general, el porcentaje de brotación o prendimiento de las varetas fue muy alto (más del 90\%) y no hubo grandes diferencias entre los patrones. El prendimiento inició un poco más rápido en los injertos sobre patrón de guayaba (Figura 1a). A los 19 ddi, el $70 \%$ de las varetas ya estaban con hoja y un $20 \%$ con brotes, mientras que en los injertos sobre cas criollo, solo había un $10 \%$ de varetas con hoja. A los 26 ddi, se completó la brotación, con formación de nuevas hojas en un $100 \%$ de las varetas de R9-33 en la mayoría de los patrones sobre los que se injertó, excepto sobre el cas criollo con solo un $90 \%$ de varetas brotadas (Figura $1 \mathrm{~b}$ ).

Independientemente del patrón, la técnica de injertación usada (con la vareta preparada previamente), permitió un alto porcentaje de brotación o prendimiento, lo cual, facilitó la reproducción asexual en guayaba. Existen otras técnicas de reproducción asexual probadas en guayaba: por estacas, acodos o in vitro, en las cuales, se requieren condiciones más cuidadosas de manejo (Dantas et al. 1999, González et al. 2002, Pérez et al. 2002, Ramírez et al. 2003, Vilchez et al. 2004).
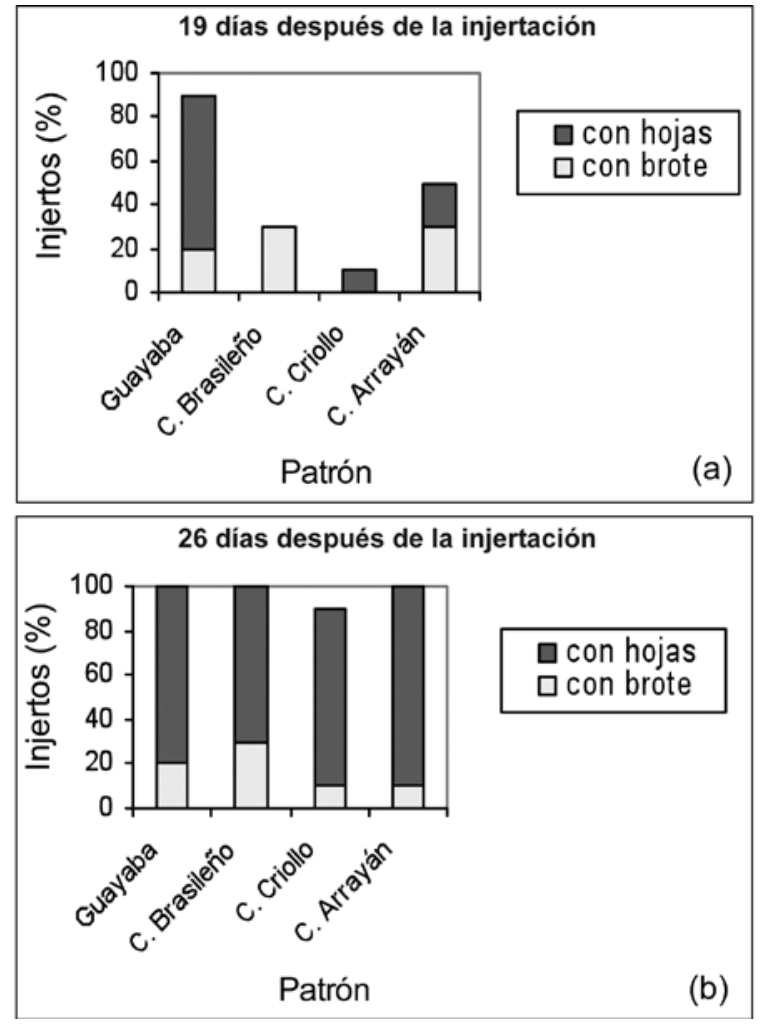

Figura 1. Porcentaje de injertos con hojas y brote en guayaba R9-33 sobre cinco patrones. a) Injertos a los 19 días después de la injertación y b) Injertos a los 26 días después de la injertación (ddi). Guápiles, Limón, Costa Rica. 2007.

\section{Crecimiento del injerto}

A los 75 ddi, se observaron diferencias en el número y en el crecimiento de las ramas de guayaba R9-33, según el patrón sobre el que se injertó. En la vareta sobre el patrón de guayaba criolla, se obtuvieron más ramas $(2,80)$, mientras que sobre arrayán un menor número $(1,70)$. Además, la longitud de la rama más larga de guayaba R9-33 fue superior sobre patrón de guayaba con respecto a los otros patrones 75 ddi (Cuadro 1).

En el tallo de los patrones de cas brasileño y criollo, se observó mayor peso de ramas (75 ddi). Esas ramas que cuando crecen son llamadas "chupones" por su dominancia apical, deben podarse del patrón como una práctica posterior al injerto, para cuidar el crecimiento de las ramas de R9-33 (Cuadro 1). En 
Cuadro 1. Número y longitud de la rama más larga de R9-33 y peso de ramas podadas en el patrón. Guápiles, Limón, Costa Rica. 2007.

\begin{tabular}{lccl}
\hline $\begin{array}{c}\text { Tratamiento } \\
\text { (patrón) }\end{array}$ & $\begin{array}{c}\text { Número de } \\
\text { ramas }\end{array}$ & $\begin{array}{c}\text { Longitud } \\
\text { de la rama } \\
(\mathbf{c m})\end{array}$ & $\begin{array}{c}\text { Peso de } \\
\text { las ramas } \\
(\mathbf{g})\end{array}$ \\
\hline 54 días después de injertada & \multicolumn{2}{l}{} \\
1. Guayaba & $1,50 \mathrm{a}^{*}$ & $11,70 \mathrm{a}$ & - \\
3. Cas brasileño & $1,70 \mathrm{a}$ & $6,30 \mathrm{bc}$ & - \\
4. Cas criollo & $1,55 \mathrm{a}$ & $4,50 \mathrm{c}$ & - \\
5. Arrayán & $1,50 \mathrm{a}$ & $8,05 \mathrm{~b}$ & - \\
\hline 75 días después de injertada & & \\
1. Guayaba & $2,80 \mathrm{a}$ & $18,80 \mathrm{a}$ & $3,27 \mathrm{~b}$ \\
3. Cas brasileño & $2,10 \mathrm{ab}$ & $10,15 \mathrm{~b}$ & $19,85 \mathrm{a}$ \\
4. Cas criollo & $2,22 \mathrm{ab}$ & $7,33 \mathrm{~b}$ & $25,00 \mathrm{a}$ \\
5. Arrayán & $1,70 \mathrm{~b}$ & $11,60 \mathrm{~b}$ & $9,72 \mathrm{~b}$ \\
\hline
\end{tabular}

*Medias con letra diferente en la misma columna, difieren según prueba Duncan $(\mathrm{P} \leq 0,05)$.

ambos patrones, hubo mucho follaje en el momento de la injertación, lo que afecta la cantidad y el vigor de los brotes, en contraposición con el crecimiento deseado de las ramas del injerto.

\section{Plantación definitiva}

El peso de las ramas podadas en los patrones y conocidas como chupones, fue diferente y considerablemente mayor en el caso de guayaba tratada con nematicida, tal vez, por mejor vigor, como una respuesta al tratamiento químico sobre las raíces (Cuadro 1, Figura 2). La poda de ramas (deschupona) en los patrones es una práctica que se hace en el vivero y con más intensidad en el campo para evitar que se pierda el injerto, por ese motivo entre menos ramas desarrolle el patrón, más ventajoso es su manejo.

Con respecto a la copa de guayaba R9-33, el número y la longitud de las ramas 135 dds, varió según los diferentes patrones (Cuadros 2, 3). La copa de R933 sobre los patrones de guayaba con y sin nematicida presentó un promedio de 13 y 17 ramas respectivamente (Cuadro 2). Dichas ramas sumaron (longitud acumulada) 662 y 937 metros de largo (Cuadro 3), lo cual es evidencia de más biomasa en la copa de esos

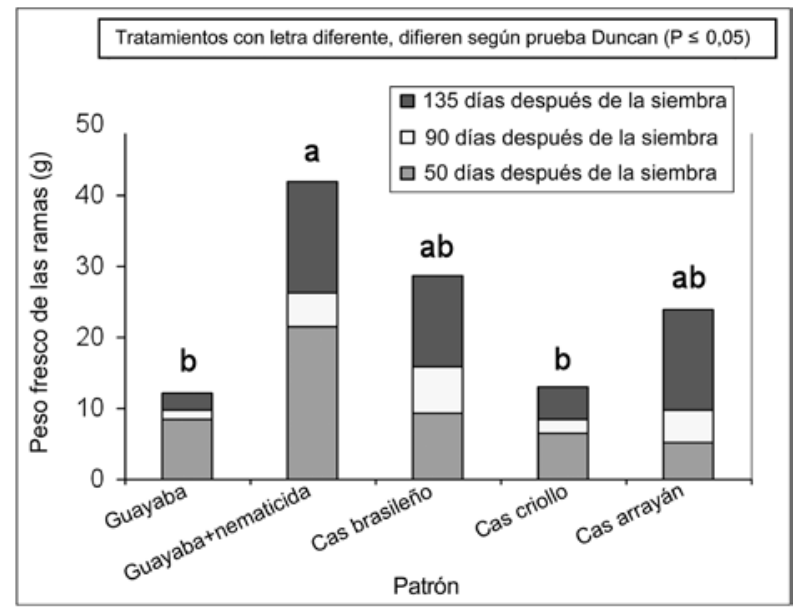

Figura 2. Peso fresco de ramas podadas a los patrones hasta los 135 días después de la siembra (dds). Guápiles, Limón, Costa Rica. 2007.

Cuadro 2. Número de ramas de guayaba R9-33 injertada en cuatro patrones en el campo. Guápiles, Limón, Costa Rica. 2007.

\begin{tabular}{lccc}
\hline & \multicolumn{3}{c}{ Días después de la siembra } \\
\cline { 2 - 4 } Tratamiento (patrón) & $\mathbf{5 0}$ & $\mathbf{9 0}$ & $\mathbf{1 3 5}$ \\
\hline 1. Guayaba & $5,80 \mathrm{a} *$ & $12,80 \mathrm{a}$ & $17,40 \mathrm{a}$ \\
2. Guayaba + nematicida & $4,00 \mathrm{~b}$ & $10,00 \mathrm{~b}$ & $13,62 \mathrm{~b}$ \\
3. Cas brasileño & $3,10 \mathrm{bc}$ & $3,90 \mathrm{c}$ & $5,60 \mathrm{c}$ \\
4. Cas criollo & $2,38 \mathrm{c}$ & $3,62 \mathrm{c}$ & $3,88 \mathrm{~cd}$ \\
5. Arrayán & $2,30 \mathrm{c}$ & $2,40 \mathrm{c}$ & $2,80 \mathrm{~d}$ \\
\hline
\end{tabular}

*Medias con letra diferente en la misma columna, difieren según prueba Duncan $(\mathrm{P} \leq 0,05)$.

árboles. Por el contrario, se pudo observar que ambas variables (número y longitud acumulada de las ramas) son indicadoras de una escasa producción de biomasa en los árboles de la R9-33 sobre cas criollo y sobre arrayán (Cuadros 2, 3).

Diferencias en el crecimiento de tres tipos de guayaba sobre cas (Psidium friedrichsthalianum) fueron observados por Marín et al. (2000), debido a la interacción de las características propias de la combinación copa y patrón. 
Cuadro 3. Promedios de longitud de acumulada ( $\mathrm{cm}$ ) de ramas de guayaba R9-33, según el patrón, en el campo. Guápiles, Limón, Costa Rica. 2007.

\begin{tabular}{lccc}
\hline & \multicolumn{3}{c}{ Días depués de la siembra } \\
\cline { 2 - 4 } Tratamiento/patrón & $\mathbf{5 0}$ & $\mathbf{9 0}$ & $\mathbf{1 3 5}$ \\
\hline 1. Guayaba & $193,00 \mathrm{a}^{*}$ & $464,60 \mathrm{a}$ & $937,80 \mathrm{a}$ \\
2. Guayaba + nematicida & $139,13 \mathrm{~b}$ & $345,63 \mathrm{~b}$ & $662,25 \mathrm{~b}$ \\
3. Cas brasileño & $58,50 \mathrm{c}$ & $104,90 \mathrm{c}$ & $226,00 \mathrm{c}$ \\
4. Cas criollo & $37,00 \mathrm{c}$ & $67,00 \mathrm{c}$ & $148,88 \mathrm{~cd}$ \\
5. Arrayán & $42,70 \mathrm{c}$ & $60,60 \mathrm{c}$ & $93,30 \mathrm{~d}$ \\
\hline
\end{tabular}

* Medias con letra diferente en la misma columna, difieren según prueba Duncan $(\mathrm{P} \leq 0,05)$.

En la guayaba R9-33 injertada sobre arrayán, es sorprendente el escaso vigor o producción de biomasa. Este patrón ejerció un efecto enanizante sobre la copa (Cuadros 2, 3). Se observó que los patrones de cas tenían un sistema radical muy reducido. Aunque no se pudo cuantificar, fue más acentuado en arrayán, en relación con los patrones de guayaba, que sí tenían un sistema radical mucho más largo y profuso.

En este trabajo los injertos fueron afectados por la acción o conformación del patrón en su sistema radical.

La acción selectiva del patrón respecto a determinados elementos nutritivos puede ser distinta a las exigencias manifestadas por el injerto. Estos cambios en la naturaleza y en la intensidad de la nutrición se manifiestan por la diferencia de vigor y en consecuencia, por el desarrollo que provocan. El patrón, de acuerdo con su propio tipo de sistema radical y con sus características genéticas puede inducir árboles de diferente capacidad de desarrollo de la parte aérea, bien sea disminuyéndola o aumentándola, en comparación con la que tuvieran si se encontraran sobre sus propias raíces. Es decir, que se establece una interacción que induce cambios en el comportamiento de los materiales que conformaron la unión (Avilán 2007). Ese resultado podría tener un uso positivo, ya que por su efecto enanizante sobre guayaba, el patrón de arrayán podría ser considerado cuando se requiera usar muy altas densidades, incluyendo el sistema de setos o si se valora la siembra de guayaba en macetas para uso en jardines con espacio reducido o la siembra en casa de mallas si se piensa en la exportación a los Estados
Unidos. También su uso podría considerarse en aquellos casos en los que se requiera atenuar el crecimiento de algún material muy vigoroso.

\section{Número de flores y frutos}

El número de flores y frutos en las plantas de guayaba R9-33 sobre los patrones de guayaba criolla fue muy superior con respecto a los casos en que se usó cas como patrón, tanto a los 135 como a los 250 dds. La producción de flores y frutos en las plantas de R933, no fue diferente entre los patrones de cas utilizados (Cuadro 4).

Cuadro 4. Número de flores (pares) y de frutos en guayaba R9-3, según el patrón, en el campo. Guápiles, Limón, Costa Rica. 2007.

\begin{tabular}{|c|c|c|c|}
\hline \multirow{3}{*}{ Tratamiento/patrón } & \multicolumn{3}{|c|}{ Días depués de la siembra } \\
\hline & \multicolumn{2}{|c|}{135} & 250 \\
\hline & $\begin{array}{c}\text { Número } \\
\text { flores }\end{array}$ & $\begin{array}{l}\text { Número } \\
\text { de frutos }\end{array}$ & $\begin{array}{l}\text { Número } \\
\text { de frutos }\end{array}$ \\
\hline 1. Guayaba & $7,80 \mathrm{a}^{*}$ & $1,40 \mathrm{a}$ & 18,70 a \\
\hline 2. Guayaba + nematicida & $8,12 \mathrm{a}$ & $1,00 \mathrm{a}$ & $16,50 \mathrm{a}$ \\
\hline 3. Cas brasileño & $3,40 \mathrm{ab}$ & $1,80 \mathrm{a}$ & $8,40 \quad \mathrm{~b}$ \\
\hline 4. Cas criollo & $2,50 \mathrm{~b}$ & $2,25 \mathrm{a}$ & $8,38 \mathrm{~b}$ \\
\hline 5. Arrayán & $2,30 \mathrm{~b}$ & $1,80 \mathrm{a}$ & 5,60 b \\
\hline
\end{tabular}

* Medias con letra diferente en la misma columna, difieren según prueba Duncan $(\mathrm{P} \leq 0,05)$.

La producción de frutos de R9-33, sobre patrones de guayaba, en ambas evaluaciones, se puede considerar como un rendimiento acorde con el número y el largo de sus ramas. Por el contrario, la baja producción de guayaba sobre los patrones de cas, es consecuencia de la baja arquitectura y poco follaje en la planta, situación que reduce la producción. La baja producción, debido al escaso volumen de la copa en árboles pequeños, puede ser compensado por un mayor número de árboles por hectárea. Además, permite asegurar una elevada eficiencia productiva (frutos $/ \mathrm{m}^{3}$ de follaje), facilita las labores de cosecha y el control de plagas y enfermedades (Avilán et al. 2003). 
El cultivo de árboles de porte enano o semienano, es muy utilizado en la actualidad, en contraposición con los de árboles de gran vigor vegetativo que caracterizó al sistema tradicional de explotación; esto parece, mucho más conveniente y económico en toda la fruticultura. Los estudios sobre el comportamiento de los frutales perennes de tipo arbóreo en el trópico, en relación con su eficiencia reproductiva lo avalan. Por eso, los sistemas de producción de altas densidades de población exigen el empleo de patrones enanizantes o en su defecto, el uso de otras prácticas que limiten el desarrollo excesivo de los árboles (Avilán 2007).

\section{Peso de fruta}

Es evidente la tendencia de un mayor peso de la fruta en los injertos sobre patrón de guayaba comparado con el peso de la fruta en los injertos sobre cas (Cuadro 5). Existe una coordinación muy estrecha entre la fuente de asimilados fotosintéticos, fundamentalmente el follaje y la raíz como destino de éstos; asimismo entre la raíz como fuente de agua y minerales y la parte aérea de la planta como destino de estos elementos (Villalobos 2001).

Con base en lo anterior se presume que el vigor que muestra la copa de guayaba R9-33, incide en frutas con mejor peso que el obtenido en las plantas sobre patrones de cas. Una planta de menos tamaño y biomasa podría ajustar sus frutos a una situación de equilibrio entre las cantidades de carbono producto de su fotosíntesis y los nutrimentos que absorbe por la raíz tal y como lo explica Villalobos (2001).

Cuadro 5. Peso de fruta de guayaba R9-33 de diferentes patrones entre 250-300 días después de la siembra. Guápiles, Limón, Costa Rica. 2007.

\begin{tabular}{lccc}
\hline Tratamiento/patrón & $\begin{array}{c}\text { Peso pro- } \\
\text { medio }(g)\end{array}$ & $\begin{array}{c}\text { Desvia- } \\
\text { ción es- } \\
\text { tandar }\end{array}$ & $\begin{array}{c}\text { Núme- } \\
\text { ro de } \\
\text { frutos }\end{array}$ \\
\hline 1. Guayaba & $338^{*}$ & 62 & 18 \\
2. Guayaba + nematicida & 310 & 60 & 20 \\
3. Cas brasileño & 235 & 77 & 26 \\
4. Cas criollo & 177 & 28 & 11 \\
5. Arrayán & 154 & 49 & 23 \\
\hline
\end{tabular}

*Sin ANDEVA.
Con respecto al peso de la fruta, el mercado mexicano, en el que se consume mucha guayaba fresca, clasifica la fruta para primera con un peso mínimo de 50 g y como "extra clase", guayaba con un mínimo de 100 g (González et al. 2002). En Brasil, la variedad Paluma es sembrada tanto para industria como para consumo fresco, la fruta se considera grande con un peso de $160 \mathrm{~g}$ (Abel et al. 2003). En nuestro país, la guayaba taiwanesa introducida por técnicos de la Misión Técnica de la República China tiene pesos que varían entre los 170 y 800 g (Román y Wann 1999).

\section{Nematodos}

En la provincia de Alajuela en Costa Rica, López y Azofeifa (1985) hicieron una identificación de los nematodos Helicotylenchus, Tylenchus, Criconemella, Trichodorus, Meloidogyne, Pratylenchus, Longidorus y Rotylenchulus en guayaba.

En las raíces de las plantas de guayaba con y sin nematicida así como en las de cas "brasileño" se encontraron poblaciones de Meloidogyne y Pratylenchus (Cuadro 6). En esos casos, las raíces y el follaje o copa de guayaba R9-33 mostraron los síntomas que Suárez y Rosales (1998) describen para plantas afectadas por Meloidogyne y consiste en la formación de agallas en las raíces, clorosis de las hojas en la parte aérea, una reducción del crecimiento, secamiento de ramas, reducción del tamaño de las hojas y cuando el ataque del nematodo es muy severo, la planta muere.

Cuadro 6. Poblaciones de nematodos, en cuatro patrones en los que se injertó la guayaba R9-33 a los 600 días después de la siembra. Guápiles, Limón, Costa Rica. 2007.

\begin{tabular}{lcc}
\hline $\begin{array}{l}\text { Tratamiento/ } \\
\text { patrón }\end{array}$ & $\begin{array}{c}\text { Meloidogyne/100 } \\
\text { gramos de raíz } \\
\text { (Probabilidad=0,40) }\end{array}$ & $\begin{array}{c}\text { Pratylenchus/100 } \\
\text { gramos de raíz } \\
\text { (Probabilidad=0,68) }\end{array}$ \\
\hline 1. Guayaba & $7000 \mathrm{a}$ & $1250 \mathrm{a}$ \\
2. Guayaba + & $11500 \mathrm{a}$ & $1750 \mathrm{a}$ \\
nematicida & $7125 \mathrm{a}$ & $1750 \mathrm{a}$ \\
3. Cas brasileño & 0 & 0 \\
4. Cas criollo & 0 & 0 \\
5. Arrayán & & \\
\hline
\end{tabular}

Medias con letras iguales en la misma columna, no difieren según prueba Duncan $(\mathrm{P} \leq 0,05)$.

*Tratamientos no incluidos en el ANDEVA. 
Investigaciones realizadas en el CENIAP demuestran que el daño causado por los hongos Fusarium oxysporum y Macrophomina phaseolina se incrementa con la presencia de Meloidogyne (Suárez y Rosales 1998). En este trabajo, las raíces de algunos patrones de guayaba sin nematicida, las cuales fueron muestreadas, presentaron un síntoma muy similar a lo que se conoce como "corchosis", descrito en las raíces de café, y que se atribuye al efecto del daño causado por dos patógenos combinados, Meloidogyne spp y Fusarium sp. (Bertrand et al. 2000).

El uso de carbofurán no tuvo un efecto muy positivo sobre los nematodos en las raíces de guayaba criolla usada como patrón (Cuadro 6). Lo anterior, pudo repercutir en la copa de R9-33 (Cuadro 3). Casassa et al. (1996) en Venezuela, concluyeron que el control químico con los nematicidas carbofurán, feamiphos y ethoprop, utilizados en dosis equivalentes de 4 y $8 \mathrm{~g}$ i.a./árbol/año no representaron una solución para reducir las pérdidas de producción en el cultivo del guayabo ocasionadas por el nematodo Meloidogyne spp. y plantearon en ese momento, probar otras dosis, otros productos o evaluar otras alternativas de control. Asimismo Cepeda et al. (2003), en el intento por recuperar árboles con daños fuertes por nematodos, concluyeron que el tratamiento a base de Furadán 5 G (40 kg/ha) no mostró efecto en la recuperación de los árboles y en algunos parámetros fue inferior al testigo sin correctivo.

En cas criollo y arrayán, no hubo presencia alguna de nematodos (Cuadro 6). Al respecto, Marín et al. (2000) no solo destacó diferencias fenológicas de tres copas de guayaba sobre cas (Psidium friedrichsthalianum) sino que citó los resultados obtenidos en Venezuela por Casassa et al. (1998), en los cuales señalan a Psidium friedrichsthalianum como una alternativa viable por su demostrada resistencia al ataque de nematodos como $M$. incognita, tanto en vivero como en campo. No obstante, con base en los datos de este estudio, no podríamos generalizar, porque como ya se mencionó, las raíces de cas brasileño ( $P$. friedrichsthalianum) si salieron infestadas con nematodos.

Relacionado con lo anterior, durante el muestreo de raíces, al arrayán no solo se le observó poca raíz, sino que su sistema radical es muy vertical con una pivotante profunda y con las raíces secundarias a $40 \mathrm{~cm}$ de profundidad, por el contario, el cas brasileño mostró un sistema radical un poco más profuso y superficial. Podríamos pensar que esa situación física (cantidad y profundidad de las raíces en el suelo) contrarresta la presencia de nematodos en esos dos patrones. Sin embargo, Casassa et al. (1998), inocularon huevos y estados juveniles del nematodo $M$. incognita en raíces de cas (Psidium friedrichshalianum) y guayaba (Psidium guajava), comprobando que en el cas usado, no hubo reproducción del nematodo.

También, se dice que la resistencia a los nematodos puede ser expresada en varios niveles: una resistencia mecánica contra la penetración que actúa retardando la colonización de los tejidos de las raícesen este sentido, Molero et al. (2006) asocian procesos mitótico de células de raíz en materiales de guayaba y cas con estos mecanismos de resistencia contra $\mathrm{Me}$ loidogyne incognita. Además, pueden ser secretadas, sustancias repulsivas o tóxicas en el tejido vegetal o en el medio exterior según el mecanismo pre-infeccioso o post-infeccioso (fitoalexinas) (Larraín 2002 ).

Se debe recalcar en términos generales que la técnica de injertación con la vareta preparada previamente, permitió un alto porcentaje de prendimiento que facilita la reproducción asexual en guayaba. Las plantas de guayaba 9-33 sobre patrón de guayaba criolla mostraron mayor vigor después de la siembra y por el contrario, se observó enanismo en la guayaba 9-33 sobre cas arrayán. Aunque se determinó la presencia de los nematodos Meloidogyne sp y Pratylenchus sp en las raíces de los patrones de cas brasileño y guayaba criolla con y sin nematicida, no se encontró nematodos en las raíces de cas criollo ni de arrayán.

\section{LITERATURA CITADA}

Abel, SJ; Tiyoko, R; Días, N; Hissayuki, H; Marines, B. 2003. El cultivo del guayabo en Brasil. In: J. Padilla, L. Reyes, E. González y M. Perales. eds. Memoria. Primer Simposio Internacional de la guayaba. Aguascalientes, México. p. 87.

Avilán, L; Soto, E; Azkue, M; Escalante, H; Rodríguez, M; Ruiz, J. 2003. Manejo de altas densidades de poblaciones en mango (en línea). CENIAP HOY no. 1, enero-abril 2003. Maracay, Aragua, Venezuela. Consultado 15 nov. 07. Disponible en www.ceniap.gov. ve/ceniaphoy/articulos/n1/texto/lavilan.htm

Avilán, L. 2007. El patrón y su importancia en la fruticultura (en línea). Investigador. FONAIAP - Centro Nacional de Investigaciones Agropecuarias. Maracay. Consultado 15 nov. 07. Disponible en http://www. ceniap.gov.ve/publica/divulga/fd58/patrones.html 4 
Bertrand, B; Núñez, C; Sarah, J. 2000. Disease complex in coffee involving Meloidogyne arabicida and Fusarium oxysporum. Plant Pathology (2000) 49:383-388.

Casassa, M; Matheus, J; Crozzolli, R; Casanova A. 1996. Control químico de Meloidogyne spp. en el cultivo de guayabo (Psidium guajava L) en el Municipio Mara del Estado Zulia, Venezuela. Rev. Fac. Agron. (LUZ) 1(13):303-312.

Casassa, A; Crozzolli, R; Matheus, J; Bravo, V; Marin, M. 1998. Efecto del nematodo agallador (Meloidogyne incognita) sobre el crecimiento del guayabo (Psidium spp.) en vivero. Nematol. Medit. 26:237-242

Cepeda, M; González, E; Gallegos, G; Padilla, J; Reyes, L; Perales, M; Reyes, H. 2003. El nematicida Ditera (Myrothecium verrucaria). Una alternativa biológica para el control de nematodos en guayaba. In: J. Padilla, L. Reyes, E. González y M. Perales. eds. Memoria. Primer Simposio Internacional de la guayaba. Aguascalientes, México. p. 222-228.

Dantas, A; Dutra, L; Kersten, E. 1999. Influencia do etefon e do tipo de estaca no enraizamiento de goiabeira (Psidium guajava L). Rev. Bras. de Agrociencia 5 (1):19-21.

González, E; Padilla, J; Reyes, L; Perales de La Cruz, M; Esquivel, F. 2002. Guayaba. Su cultivo en México. Libro técnico No. 1. INIFAP. Campo Experimental Pabellón. Aguas Calientes. México. 182 p.

Larraín, A. 2002. Nematodos en raíces de portainjertos de frutales de Carozo (Prunus sp). Residencia presentada como parte de los requisitos para optar al título de Ingeniero Agrónomo (Tesis). Facultad de Agronomía e Ingeniería Forestal. Departamento de Fruticultura y Enología. Pontificia Universidad Católica de Chile. Santiago. Chile. 57 p.

López, R; Azofeifa, J. 1985. Nematodos fitoparásitos asociados con frutales en algunos cantones de la provincia de Alajuela. Agronomía Costarricense 9(2):193-195.

López, PG; López, PJ. 1995. Introducción al Micro SAS. Aplicación al análisis de experimentos. Unidad de Informática y Bioestadística. Centro Agronómico de Investigación y Enseñanza (CATIE). Turrialba. Costa Rica. 119 p.

Marín, M; Casassa, A; Rincón, A; Labarca, J; Hernández, Y; Gómez, E; Viloria, Z; Bracho, B; Martínez, J. 2000. Comportamiento de tipos de guayabo (Pidium guajava
L.) injertados sobre Psidium friedrichsthalianum. Revista Facultad Agronomía La Universidad del Zulia (LUZ) 17:384-392.

Molero, T; Molina, J; Barrios, Casassa, A. 2006. Proceso mitótico en células radicales de Psidium spp. tolerantes, susceptibles, y resistentes a Meloidogyne incognita en el estado Zulia, Venezuela. Bol. Centro Invest. Biol. 40(1):41-56.

Monteverde, E. 1989. Evaluación del Naranjo "Valencia" sobre diez Patrones en valles Altos de Carabobo - Yaracuy. II. Calidad del Fruto y Recomendaciones. Maracay Venezuela. FONIAP Divulgación $N^{\circ} 32$ Julio-Diciembre. 5 p.

Perales, MA; Padilla, J.; González, E; Reyes, HR. 2005 Manual para la producción integral del cultivo de la guayaba. Pabellón de Arteaga. Secretaría de Agricultura, Ganadería, Desarrollo Rural, Pesca y Alimentación. Instituto Nacional de Investigaciones Forestales, Agrícolas y Pecuarias. Centro de Investigación Regional Norte Centro. Campo Experimental Pabellón, Aguas Calientes, Zacatecas, México. 79 p.

Pérez, A; Nápoles, L; Concepción, O; Trujillo, R. 2002. Multiplicación in vitro de brotes de guayaba (Psidium guajava $\mathrm{L}$ ) var. Enana roja cubana EEA 18-40 obtenidos a partir de semillas. Cultivos Tropicales 23(3):57-61.

Ramírez, A; Cruz, N; Franchialfaro, O. 2003. Uso de bioestimulantes en la reproducción de guayaba (Psidium guajava L.) mediante el enraizamiento de esquejes. Cultivos Tropicales 24(1):59-63.

Román, R; Wann, J. 1999 Cultivo de la Guayaba. Misión Técnica de la República China (Taiwán). MAG, IDA. Cañas. Costa Rica. mimeografiado. 21 p.

Suárez, Z; Rosales, L. 1998. Nematodos asociados a los frutales y su control. In: frutales perennes. CENIAP. IIA. Maracay Venezuela. FONIAP DIVULGA No. 59. 7 p.

Vilchez, J; Bracho, I; Arenas, N; Marín, M; Martínez, L. 2004. Respuesta a la técnica de acodo aéreo en plantas de guayabo (Psidium guajava $\mathrm{L}$ ) tolerantes al nematodo Meloidogyne incognita. Rev. Fac. Agron. (LUZ) 21 Supl. 1:22-27.

Villalobos, E. 2001. Fisiología de la producción de los cultivos tropicales. Procesos Fisiológicos Básicos. Fascículo I. San José, Costa Rica. Editorial de la UCR. 228 p. 